Recepción: 08 / 01 / 2017

Aceptación: 10 / 03 / 2017

Publicación: 15 / 05 / 2017

\title{
Razonamientos de unificación de energía solar activa en arquitectura
}

Reasoning for unification of active solar energy in architecture

\section{Raciocínio para unificação de energia solar ativa em arquitetura}

\author{
Alexis J. Macías-Loor ${ }^{\mathrm{I}}$ \\ alexismaloor@gmail.com
}

Alexis J. Macías-Mendoza II alexismacias80@gmail.com

Correspondencia: alexismaloor@gmail.com

\footnotetext{
I Magister en Gerencia Educativa, Arquitecto, Universidad Laica Eloy Alfaro de Manabí, Manta, Ecuador.

${ }^{\text {II }}$ Master Universitario en Planeamiento Urbano y Territorial, Arquitecto.
} 



\section{Resumen}

La problemática energética mundial induce a la necesaria inclusión de medidas de eficiencia energética en edificaciones y ciudades. No obstante, ello no es suficiente si el objetivo es prescindir definitivamente de las energías fósiles, así como minimizar el impacto a la naturaleza como consecuencia de la obtención energética. Por ello, la inclusión de alternativas de autoabastecimiento en las propias edificaciones es fundamental. El sol es un recurso gigantesco, especialmente en zonas de latitudes medias y enfáticamente ecuatoriales. Este trabajo revisa antecedentes documentales de integración de energía solar activa desde la perspectiva de la arquitectura, recopilando antecedentes históricos, tecnologías disponibles en concordancia con las demandas, así como consideraciones tecnológicas que deberán tenerse en cuenta en las edificaciones. Se enuncian, además, los postulados recientes respecto a la integración arquitectónica como aspectos funcionales y morfológicos. A partir de este análisis se proponen niveles de integración arquitectónica. De las condiciones y posibilidades analizadas depende que, al ser considerados en el diseño, los colectores solares sean eficientes en producción y en concordancia con la arquitectura.

Palabras clave: arquitectura bioclimática; colector solar; calefacción solar; célula solar; edificio solar.

\section{Abstract}

The global energy problem induces the necessary inclusion of energy efficiency measures in buildings and cities. However, this is not enough if the objective is to permanently dispense with fossil fuels, as well as to minimize the impact on nature as a result of obtaining energy. Therefore, the inclusion of self-supply alternatives in the buildings themselves is fundamental. The sun is a gigantic resource, especially in areas of mid-latitudes and emphatically equatorial. This work reviews documentary background of integration of active solar energy from the perspective of architecture, compiling historical background, available technologies in accordance with the demands, as well as technological considerations that should be taken into account in buildings. In addition, the recent postulates regarding architectural integration as functional and morphological aspects are stated. From this analysis, levels of architectural integration are proposed. Of the conditions and possibilities analyzed, it depends that, when 
considered in the design, the solar collectors are efficient in production and in accordance with the architecture.

Keywords: bioclimatic architecture; solar collector; solar heating; solar cell; solar building.

\section{Resumo}

O problema energético global induz a inclusão necessária de medidas de eficiência energética em edifícios e cidades. No entanto, isso não é suficiente se o objetivo é dispensar permanentemente os combustíveis fósseis, bem como minimizar o impacto sobre a natureza como resultado da obtenção de energia. Portanto, a inclusão de alternativas de auto-abastecimento nos próprios edifícios é fundamental. O sol é um recurso gigantesco, especialmente em áreas de latitudes médias e enfaticamente equatorial. Este trabalho analisa o histórico documental da integração da energia solar ativa a partir da perspectiva da arquitetura, compilando o contexto histórico, as tecnologias disponíveis de acordo com as demandas, bem como as considerações tecnológicas que devem ser levadas em conta nos edifícios. Além disso, os postulados recentes sobre a integração arquitetônica como aspectos funcionais e morfológicos são apresentados. A partir dessa análise, os níveis de integração arquitetônica são propostos. Das condições e possibilidades analisadas, depende que, quando considerados no projeto, os coletores solares sejam eficientes na produção e de acordo com a arquitetura.

Palavras chave: arquitetura bioclimática; coletor solar; aquecimento solar; célula solar; construção solar.

\section{Introducción}

Este trabajo forma parte de los proyectos de investigación "Método de certificación de la construcción sustentable de viviendas" apoyado y financiado por la Dirección de Investigación de la Universidad de Cuenca (DIUC), y "Determinación de potencial de integración arquitectónica de captación solar activa en tipología constructiva de baja altura en centro patrimonial de ciudades andinas. Caso de Estudio Cuenca, Ecuador", del Centro de Investigaciones de la Facultad de Arquitectura de la Universidad de Cuenca. 
La arquitectura debe cobijar las actividades frente al clima y los agentes externos, pero también debe aprovechar algunas condiciones naturales, como la radiación solar. Por ejemplo, a través de la captación pasiva por ventanas y muros envolventes del edificio, es factible regular la temperatura, otorgar iluminación y desinfectar ambientes. No obstante, se deben controlar consecuencias adversas como el encandilamiento, la acumulación o pérdidas excesivas en ciertos periodos, debido a que los aportes solares varían según las estaciones del año u horas del día. Las aberturas implican ganancias térmicas y lumínicas por asoleamiento, pero también potenciales fugas si están dispuestas en costados con escasa exposición solar (Besser, Rodrigues y Bobadilla, 2012; Guillén et al., 2014).

Existen además requerimientos de iluminación o climatización de edificios, en horarios nocturnos o espacios inaccesibles, que son suplidos con equipos que consumen combustibles, mayormente de origen fósil. Como alternativa, estos requerimientos pueden ser salvados con tecnologías que capturan la energía solar y la aportan a distancia o en momentos diferidos. Esto se conoce como captación activa, que consiste en recibir la radiación solar y convertirla en otras formas de energía, mediante colectores térmicos (de calor) o fotovoltaicos (de electricidad). No obstante, los servicios energéticos en un edificio deben estar primeramente supeditados a medidas de eficiencia pasivas, para reducir los requerimientos activos.

Una de las alternativas más provisorias para las futuras comunidades es el abastecimiento energético desde los propios edificios y ciudades, considerando que estas ya son intervenciones e invasiones humanas con mayor o menor impacto en el contexto natural. La irradiación solar que recae sobre las ciudades se desperdicia en su mayor parte, a veces incluso con efectos nocivos al incrementar el efecto "isla de calor". El autoabastecimiento desde energías renovables implica evitar emanaciones de $\mathrm{CO} 2$, así como se prescinde de invadir otras zonas externas a las ciudades para generación energética. La captación activa puede aportar a otros recintos o edificios más alejados, mediante almacenamiento, transmisión o redes colectivas inteligentes o Smart Grid (EREC, 2010; Gook-hwan y Eximbank, 2013; IEA, 2009; Lund, 2012; Mikkola y Lund, 2014). Este trabajo revisa la incorporación del sol en la arquitectura y la manera como debería integrarse los dispositivos requeridos como nuevos componentes arquitectónicos; además, se definen algunos lineamientos que el arquitecto debe considerar para lograr resultados técnicos y arquitectónicos eficientes. 


\section{Antecedentes}

\section{Desarrollo histórico}

La arquitectura recurre al sol desde tiempos prehistóricos. Registros de edificaciones griegas de 2.500 años atrás sugieren medidas de asoleamiento por la escasez de combustible natural (Vázquez, 1999). Incluso los atenienses reglamentaron el corte de olivos para leña y codificaron condiciones para que las viviendas tuvieran una adecuada captación solar y masa térmica. Portales, patios, aleros y vanos fueron proporcionados según la latitud de las ciudades griegas (alrededor de $40^{\circ}$ norte), para evitar la radiación solar durante los meses calurosos y aprovecharla durante el invierno. Por otro lado, en la arquitectura se han utilizado diversas estrategias bioclimáticas pasivas para disminuir el consumo energético al aprovechar la energía solar. Por ejemplo, con la utilización de masa para acumulación térmica se ha logrado regular la temperatura para un mejor aprovechamiento. En climas más cálidos, en cambio, la estrategia es evitar el ingreso de radiación solar directa para disminuir el sobrecalentamiento. En edificaciones romanas para baño se observan ventanales extensos orientados hacia el sur para mejoramiento térmico (Zhu et al., 2011).

En el siglo XVIII, la explotación del carbón lleva a acondicionar edificaciones a través de la combustión fósil, lo cual provoca un alejamiento de las estrategias pasivas. Los principios de captación pasiva vuelven a la palestra a finales del siglo XX por el colapso del sistema energético. La mayoría de los países desarrollados establecen regulaciones de edificación que fomentan el aprovechamiento solar pasivo para mitigar los crecientes consumos energéticos, mediante estudios ambientales que promueven la exposición solar de construcciones y aberturas, aunque su aplicación es muy variada y aún incipiente.

\section{Antecedentes de colectores solares térmicos}

Con la generalización del vidrio, Horace de Sauserre analiza, en 1767, el efecto invernadero al describir el comportamiento térmico interior de carruajes vidriados bajo exposición solar, los cuales alcanzan elevadas temperaturas en un tiempo corto (Zhu et al., 2011). Luego, en Inglaterra, las casas empiezan a incorporar invernaderos (Menayo, 2010; Vázquez, 1999). Los primeros calentadores de agua solar fueron simples tanques metálicos expuestos al sol. En Baltimore, Clarence Kemp evoluciona este sistema en 1891, alcanzando temperaturas superiores 
a las requeridas para un baño confortable, por lo que fueron los primeros colectores solares térmicos (ST) (Perlin, 2013). En España existe registro de la primera patente en 1921, y de su comercialización y exportación a finales de los años treinta, para reemplazar calentadores de carbón (Lorenzo, 2004). La industria se expande con relativa rapidez hasta la irrupción del petróleo y el gas a muy bajo costo, con redes urbanas de distribución de gas y calentadores subsidiados por las empresas. Esto limita el crecimiento del sector solar térmico, que resurge en los años setenta por la escalada del petróleo (Vázquez, 1999).

En este periodo surgen varios programas públicos que fomentan el uso de colectores solares en edificación para agua caliente sanitaria (ACS) y calefacción (Gajbert, 2008; IEA y SHC, 1977; Kalogirou, 2004; Suter, Letz y Weiss, 2003). Con la tecnología actual, la captación térmica activa alcanza altas eficiencias, pero está limitada por la intermitencia y fluctuación solar. En regiones estacionales extremas el problema se acentúa al incrementar el desbalance de irradiación disponible frente a las demandas de edificios. La ausencia solar nocturna implica la necesidad de almacenamiento. Pero oscilaciones estacionales considerables requieren un mayor aislamiento, un gran volumen de almacenamiento y la eficiencia se ve reducida por la poca producción en época de mayor demanda.

\section{Surgimiento de la captación fotovoltaica en edificios}

Luego de la introducción de la energía fotovoltaica (PV) hacia fines de la década de los setenta, a partir de la industria espacial e instalaciones remotas, el Departamento de Energía de Estados Unidos comienza a promover aplicaciones domésticas. Se instala la Estación Experimental Residencial Southwest en Nuevo México y en el campus Las Cruces de la Universidad de Nuevo México. El primer proyecto residencial emblemático es la Carlislie House (1980) en Massachusetts, siendo la casa particular pionera en adopción de placas en su cubierta y con enlace a la red pública, entregando excedentes y comprando electricidad en ausencia de irradiación, con una capacidad de producción de 7,5 kWp mediante módulos de sílice cristalino. En 1983, la Boston Edison Company encarga la residencia Impact 2000 House que integra eficiencia pasiva, estrategias activas y la instalación de 4,5 kWp det placas integradas. En 1993, el Departamento de Energía de Estados Unidos inicia el programa de investigación

"Oportunidades para edificaciones de Estados Unidos para PV". El desarrollo comercial de la 
tecnología se ve eclipsado por Europa y Japón, que miran alternativas energéticas con mayor urgencia.

A mediados de los años ochenta, en Japón, Sanyo desarrolla la casa Kansai electrices PV Demo House. A principios de los años noventa, en Alemania se pone en marcha el programa piloto denominado "1.000 techos fotovoltaicos". Uno de los casos iniciales importantes es una vivienda en Helmstedt que integra 80 paneles PV configurados para integrarse en techumbre. En 1993, el Ministerio Bávaro del Ambiente inaugura su edificio, el cual integra en su fachada células de sílice amorfo y filtros solares constituidos por celdas de sílice cristalino. Otro ejemplo de importancia en Alemania es el edificio prototipo Stadtwerke Aachen (STAWAG), un servicio municipal que posee en su fachada sur $50 \mathrm{~m} 2$ de vidrio con celdas PV policristalinas ubicadas entre el laminado de dos cristales templados (Bolinger y Wiser, 2002). En Suiza, la Escuela Politécnica de Lausanne (EPFL) ejecuta 14 pabellones donde se muestran distintas alternativas de instalaciones PV integradas a edificación. Un gran proyecto con PV integrado en fachada es desarrollado por la empresa Solution \& Atlantis para Scheidegger Metallbau en Kirchberg, en el año 1992. En Inglaterra, el surgimiento de PV es levemente posterior, la primera instalación fue en la fachada de la University of Northumbria en Newcastle; otra instalación importante en 1995 de $10 \mathrm{kWp}$ es el Center Alternative for Technology en Gales. En el resto de Europa también se suceden al final del siglo XX importantes instalaciones como el plan para 200.000 cabañas en zonas remotas de Finlandia, la mayoría vacacionales, que carecen de conexión por estar lejanas de las redes eléctricas (Little, 1995). En este contexto, entre 1990 y 1995 se desarrolla la Task 16 de la Agencia Internacional de la Energía, que propone los primeros lineamientos para la adopción de colectores solares en la arquitectura (IEA Solar Heating y Cooling Program Task 16, 1995).

\section{Método}

Se definen los lineamientos teóricos que el arquitecto debe considerar para lograr resultados técnicos y arquitectónicos eficientes tantos tecnológicos como funcional y estéticamente, para incluir sistemas solares activos en edificaciones de manera adecuada. A partir del análisis de antecedentes y su evolución técnica, se aborda la connotación de la aplicación tecnológica en el marco de la arquitectura. Para ello se estudian distintos planteamientos teóricos aún en discusión, 
enfatizando en requerimientos necesarios en la envolvente para el despliegue de captadores solares.

En primera instancia, a partir de estudios y normas se analizan las opciones tecnológicas y las posibilidades de despliegue en distintas localizaciones de la envolvente, así como paralelismos respecto a la volumetría; se describen las potencialidades de abastecimiento, analizando a la vez distintas conceptualizaciones de "integración arquitectónica". A partir de ello, se enuncian las primeras conceptualizaciones teóricas básicas emitidas por Kaan y Reijenga (2004) a partir de una sesión de investigación inicial de fotovoltaicos en edificación, por la Agencia Internacional de Energía (IEA Solar Heating y Cooling Program Task 16, 1995). Luego se analiza el relevamiento de productos comerciales tomando como referente principal la investigación de Munari (2009) quien define posibilidades morfológicas de adaptación arquitectónica. Luego, se considera el estudio internacional efectuado en la red de investigación creada entre varios países por la Agencia Internacional de la Energía en la Task 41 del programa de Calefacción y Refrigeración Solar (Wall et al., 2012). Esta revisión permite decantar las teorías de los autores citados, para proponer niveles de integración señalados por el presente planteamiento teórico. Por último, se vislumbra la potencialidad a futuro, así como las barreras actuales que se perciben como trabas, como consecuencia de la disponibilidad tecnológica y de herramientas proyectuales y, fundamentalmente, por el desconocimiento disciplinar de los arquitectos, barrera que debe ser salvada a través de la difusión de las tecnologías en la arquitectura para su consideración efectiva en la proyectación.

\section{Resultados}

\section{Captación solar activa y arquitectura}

La incorporación de sistemas de energía solar activa en edificios consiste en disponer colectores solares para cubrir demandas energéticas propias, e incluso para contribuir con demandas externas urbanas. Para ello se disponen equipos de recolección solar en la envolvente (techumbres o fachadas), a fin de obtener irradiación, transformarla en energía útil, almacenarla y distribuirla con conexiones al lugar y momento de existencia de demanda. 


\section{Criterios funcionales}

Las tecnologías solares activas se dividen en térmicas, fotovoltaicas, o una combinación de ellas (híbridas), con capacidad de aportar a las distintas demandas operativas de un edificio. Las edificaciones poseen diversos usos, ya sea para residencia, oficinas, colegios, comercios, hospitales; con ello distintas necesidades térmicas (calor o frío) y eléctricas (fuerza motriz, iluminación), dependientes de condiciones programáticas, climáticas y solares. En el proyecto de edificación debe dimensionarse el abastecimiento solar en relación con los requerimientos funcionales, definiendo una integración entre medidas pasivas y tecnología PV, ST o ambas.

La alternativa de colectores híbridos, con capacidad de generar electricidad y temperatura en una misma superficie (Chow, 2010) está limitada por una adecuada correlación de demandas, que se puede suplir además con paneles de un solo tipo como complemento. Una opción es cubrir la máxima demanda térmica, y si ello implica obtención de excedentes eléctricos pueden ser entregados a la red urbana. Al contrario, se puede dimensionar solo para abastecer la máxima demanda térmica individual de la edificación, ya que producir excedentes térmicos no es recomendable, considerando que el almacenamiento o venta externa de calor implica pérdidas cuantiosas.

Las energías térmica y eléctrica no deben valorarse igual en correspondencia a su potencia neta, por lo que se debe tener en cuenta el concepto de exergía 1). Si consideramos la obtención de electricidad por termo-generación, un margen razonable es 3,5 unidades térmicas para obtener una eléctrica, aunque varía acorde a matriz eléctrica local. La forma más eficiente de uso de energía solar térmica es abastecer la demanda de agua caliente sanitaria o calefacción; la producción eléctrica PV debería destinarse a suplir demandas eléctricas o entregarse a la red pública. A través de la generación eléctrica PV se pueden suplir todas las demandas; no obstante, su conversión para cubrir demandas térmicas usualmente no es eficiente. Una alternativa para calefacción a partir de electricidad son las bombas de calor de alto desempeño (Coefficient of Performance, COP > 4). La refrigereciente, y aún costosa (IEA y SHC, 2015), aunque a futuro se espera que sea una opción, especialmente en lugares con grandes demandas de refrigeración en zonas con alta irradiación (Naranjo-Mendoza, Rousse y Quesada, 2013). 


\section{Capacidad volumétrica para aprovisionamiento solar}

El potencial solar de un edificio depende de sus superficies con exposición solar en concordancia con la latitud, y la trayectoria solar diaria y estacional (que modifica el ángulo de radiación respecto al plano terrestre). Mientras la localización esté más próxima a la línea ecuatorial el sol recorre a mayor altura con irradiación más vertical, por tanto, es mayor sobre las cubiertas de los edificios. En las superficies orientadas hacia la trayectoria solar, en la zona tórrida, la mayor irradiación proviene cenitalmente; en zonas meridionales en el hemisferio norte, las preponderancias poseen superficies inclinadas con orientación sur, y en el hemisferio sur hacia el norte, siendo maximizada la irradiación directa en un ángulo similar a la latitud. No obstante, en zonas más lejanas del ecuador, como por ejemplo los países nórdicos, las fachadas bien orientadas (superficies verticales) ganan importancia, especialmente para lograr irradiación en invierno, concordante con el incremento de demanda térmica, reduciendo a la vez el exceso de producción en verano.

Por esto, en latitudes medias o ecuatoriales, volumetrías de edificación mayormente horizontales, cuya área habitable es similar a la cobertura, poseen una alta potencialidad de captación solar. Mientras los volúmenes más verticales poseen una potencialidad más baja. En la franja tórrida las cubiertas reciben irradiación durante todo el año y con nula variabilidad estacional; en las fachadas es mínima (hacia el este en la mañana y oeste en la tarde), en que además inciden normalmente fragmentaciones como balcones, desniveles, vanos o circulaciones y obstrucciones del entorno (en especial edificios altos cercanos) que reducen en gran medida su capacidad, fundamentalmente en horas tempranas o tardías del día.

Estas diferencias volumétricas están relacionadas a su vez con tipologías que poseen requerimientos energéticos diferentes. Las morfologías horizontales son usualmente viviendas unifamiliares o edificios de servicios o industrias (Figura 4, diagrama C). Las morfologías verticales son desarrolladas usualmente por edificios multifamiliares de departamentos y oficinas. También las viviendas acogen actividades más regulares y demandas más constantes en el tiempo, más habitadas en periodos nocturnos e invernales en países estacionales, mientras los servicios u oficinas poseen requerimientos fundamentalmente durante el día y regulares todo el año. Lo que implica que las morfologías horizontales de servicios e industrias poseen una alta 
potencialidad de integración especialmente en la franja tórrida, mientras que en las horizontales de viviendas es media-alta; las verticales de oficinas, media-baja y de vivienda, baja (Figura 4, diagrama C). Adicionalmente, puede reconocerse una condición intermedia como bloques de vivienda, centros comerciales, servicios de oficina, hospitales, etc., con una proporción elevada de fachada (4 a 5 pisos) e intermedia en cubierta (Figura 4, diagrama B), condición que presenta un potencial relevante en latitudes medias para autoabastecerse.

\section{Integración en la edificación}

Consumir energía obtenida en el mismo lugar o muy próximamente es una condición ideal ambiental y operacionalmente, porque reduce las pérdidas por traslado a la vez que promueve la autonomía energética. En el 2013, en Estados Unidos, el 28,6 \% de generadores PV han sido instalados en edificaciones, desde residencias hasta edificios industriales. A escala mundial se estima que entre el 25 al $35 \%$ de los generadores PV corresponden a instalaciones residenciales (Rickerson y IEA, 2014).

Gran parte de los autores piensa que los colectores solares se consideran integrados arquitectónicamente cuando reemplazan elementos de recubrimiento o cumplen otras funciones de connotación arquitectónica. Instalar colectores integrados en el edificio enfrenta algunas barreras y conocimientos adicionales para los profesionales. Como la justificación económica, el involucramiento del comitente o desarrollador, datos de productos, incentivos del Gobierno, recursos instrumentales o literatura que oriente consideraciones arquitectónicas frente a captación, elementos captadores adaptables, etc. La difusión y el fomento de programas públicos o iniciativas industriales pueden impulsar este desarrollo. Otro aspecto ventajoso es la apariencia de alta tecnología y de preocupación ambiental que conlleva la presencia visible de colectores, situación que por sí sola ha motivado instalaciones sobre todo en empresas.

\section{Proyecto integrado}

Es necesario disponer de herramientas proyectuales que analicen la producción energética durante etapas tempranas de diseño, considerando por simultáneo las consecuencias del despliegue de colectores. Con procesos manuales, como el boceto o modelos físicos, se pueden estimar asoleamientos en distintas superficies y prever la posible incidencia de captación pasiva, e incluso activa, desde la perspectiva espacial y aspectual, pero no es posible cuantificar aportes 
precisos. Existen simuladores numéricos de cálculo de irradiación y algunos más avanzados que entregan la posible contribución térmica y PV. A través de fórmulas como F-Chart (Haberl y Cho, 2014) o RETScreen ${ }^{\circledR}$ (Natural Resources Canadá, 2016), el usuario puede introducir datos numéricos especificando emplazamiento, rendimiento de colectores, precios de energía y de tecnologías, los cuales de forma sencilla pueden arrojar presunciones de rendimiento, ahorro, valor futuro, etc. Sin embargo, poseen una interfaz numérica, con gráfica elemental para expresar rendimiento y sin posibilidad de interacción con software de diseño arquitectónico.

Adicionalmente están los software específicos de recolección solar, que entregan simulaciones dinámicas de alta precisión como Watsun ${ }^{\circledR}, \mathrm{SAM}^{\circledR}$ o Trnsys ${ }^{\circledR}$ (IEA et al., 2012) los cuales requieren datos completos de los dispositivos de captación y componentes del sistema, como especificaciones de almacenamiento, conversores, redes, etc.; estos entregan resultados técnicos consistentes como volumen de almacenamiento horario, capacidad de provisión, etc. La limitación existente es la fiabilidad de irradiación y demanda programada como datos de entrada, los cuales, aunque son previsiones estadísticas, difícilmente se ajustarán a la realidad de manera exacta. Si bien estas herramientas poseen una interfaz gráfica que puede reflejar el anteproyecto, no está en correlación directa con la herramienta de diseño, entonces las ediciones gráficas en los simuladores no actualizan los modelos de diseño, lo cual requiere doble trabajo o la necesidad de interexportación de modelos virtuales; además, requiere conocimientos profundos de ingeniería para calibrar los modelos.

Recientemente se ha coordinado la interacción de software de simulación solar con diseño, por ejemplo, el software Trnsys® a incluido el "Type 56" y "TRNbuild", librería que posibilita la modulación geométrica y de superficies en herramienta de arquitectura SketchUp®; no obstante, el modelo debe ser redibujado con una herramienta especial incluida específicamente para la creación de zona térmica, que no es la misma del diseño volumétrico, haciéndose necesario un "redibujado". El módulo Vasari® o Ecotect ${ }^{\circledR}$ de Autodesk permite medir de irradiación sobre superficies creadas en software BIM (Building Information Modeling) como Revit, aunque no simula el desempeño o la producción de colectores solares

Un avance más reciente es el logrado por programas de diseño arquitectónico como el BIM Archicad®, el cual ha incorporado el módulo Ecodesigner ${ }^{\circledR}$ que permite estimar demandas a la 
vez que producción solar activa térmica durante el proyecto (Zalamea León y García Alvarado, 2014). Otro software que está en desarrollo es RenewBIM, herramienta que recibe modelos tridimensionales desde BIM a través de código abierto IFC (Industry Foundation Classes), calculando la posible contribución PV al señalar una superficie como colectora (Gupta et al. , 2014). Las limitaciones de estos es ser herramientas cerradas que entregan datos generales mensuales y anuales en que pocos parámetros pueden editarse.

\section{Integración morfológica}

En relación con la disposición de colectores solares en la edificación existen diferentes posturas, por ejemplo, la norma chilena considera las situaciones que se describen a continuación, para admitir reducciones de rendimiento como consecuencia de la relación de los colectores respecto al edificio y conservar el subsidio de apoyo a la instalación (Cdt, 2007; Ministerio de Energía de Chile, 2010).

Colectores simplemente colocados: cuando se instalan elementos colectores sin relación formal, material o de color con la edificación, por lo general son el resultado de aprovechar una máxima irradiación o para evitar incidencia de sombras. El caso más recurrente son colectores térmicos con almacenamiento integrado, colectores térmicos que conllevan un mayor impacto en cuanto a su apariencia, incrementando el contraste volumétrico. Desde el punto de vista arquitectónico sería la opción menos deseable.

Colectores superpuestos en edificación: cuando las placas guardan un paralelismo con uno de los planos de la edificación (techumbre o fachada). A veces en relación con la composición y ritmos del edificio, es decir, centrado o en un eje formal, con alguna relación en color y materialidad. El colector es independiente constructivamente respecto a la envolvente, por lo que existe una doble-capa (IEA SHC Task 41, 2012b).

Colectores integrados: cuando reemplazan recubrimientos o elementos funcionalesarquitectónicos de una edificación, cumpliendo especificaciones que cualquier material de exteriores debe proporcionar, como estanqueidad y resistencia mecánica, o funcionar adecuadamente como balaustrada, filtro solar, ventanal, etc. Para ello, los colectores deben contemplarse dentro de la geometría y composición estética; deben respetar modulación y 
relación con juntas materiales y reglas compositivas, en lo posible contribuyendo positivamente a la expresión arquitectónica.

\section{Captación solar integrada}

Por otro lado, los colectores solares para integración en envolvente de edificios deben cumplir condiciones constructivas y de apariencia que frecuentemente penalizan la eficiencia de desempeño, lo que puede superarse con una mayor área de recolección, desarrollo tecnológico o reducción de costos. En general, los paneles de captación fotovoltaica y térmica para integración tienen alta variabilidad y distintas características geométricas, tecnológicas y de aspecto, por lo que no es factible generalizar.

Existen variadas posibilidades en cuanto a forma, utilidad, configuración e instalación. En primera instancia, se debe considerar también la diferenciación entre placas térmicas o fotovoltaicas que resulta en sustanciales variaciones de integración y técnica constructiva. En los del primer tipo, los fluidos se transmiten por ductos y tuberías, son más eficientes en porcentaje de aprovechamiento de radiación pero requieren almacenamiento considerable en sitio y lo más cercano a la zona de demanda; en el segundo caso, se obtiene electricidad por lo que se conectan solamente cables, son menos eficientes frente a aprovechamiento de irradiación, aunque las pérdidas eléctricas por conducción son mínimas; sin embargo, es necesario un adecuado dimensionamiento sobre todo por sobreproducción estacional o limitaciones de la red, en el caso de darse instalaciones a gran escala en edificios o barrios enteros de una zona urbana (Wegertseder et al., 2016).

Definiciones morfológicas: la relación formal de los colectores con el diseño del edificio involucra diferentes aspectos. Por ejemplo, algunos autores mencionan la integración arquitectónica como una condición especial del captador, formando parte de la edificación en función dual (IEA y SHC Task 41, 2012a; Munari, 2009). No obstante, existen algunas clasificaciones, variaciones o gradaciones respecto a calidades y la relación lograda con la edificación, las cuales se determinan a continuación. 


\section{Aplicación según tecnología}

La incorporación de sistemas de recolección solar en edificios puede variar según la ocupación. De acuerdo con el uso las actividades funcionales varían, así también las características morfológicas, constructivas y de emplazamiento urbano, además de variaciones climáticas y culturales. Las edificaciones residenciales, por ejemplo, son utilizadas de manera más continua en el tiempo que otras construcciones y mayormente en periodos nocturnos y durante clima adverso. Mientras que la ocupación de los edificios de servicios es más intermitente y, sobre todo, diurna; además, son construcciones mayores, generalmente ubicadas en la zona céntrica de la ciudad. Poseen distintos requerimientos de calefacción o refrigeración según la zona geográfica y las estrategias constructivas. A continuación, se revisan diferentes sistemas, aunque con un enfoque en modelos residenciales.

\section{Recolección térmica para agua caliente sanitaria y calefacción}

La captación solar activa residencial en Latinoamérica se ha utilizado especialmente para la instalación de sistemas térmicos para abastecer agua caliente sanitaria, impulsados en gran medida por el precio más asequible, por tratarse de instalaciones menos "tecnológicas" respecto a la PV, y por su elevada eficiencia. Los colectores típicamente utilizados son de placa plana, estos poseen alrededor de $2 \mathrm{~m} 2$ de superficie colectora y un estanque de almacenamiento de entre 120 a 1601 . El contenedor está integrado a la placa en forma cilíndrica en los sistemas más sencillos. El flujo de líquido funciona por termo-sifonamiento y traslado de temperatura. Los colectores tipo placa plana son económicos y eficientes frente a irradiación directa. En ambiente cálidosoleado tienen la capacidad de máximo aprovechamiento, no obstante, en lugares fríos y nubosos o con radiación difusa no poseen buen rendimiento, existiendo pérdidas por la propia capa absorbente a pesar de ser vidriados.

Bajo condiciones de radiación difusa, los colectores de tubos de vacío poseen mejor desempeño, propicios para clima estacional en época de invierno para contribución para calefacción y ACS, combinación denominada instalación tipo Combi System (IEA y SHC Task 26, 2000; Lamnatou et al., 2015; Suter, Letz y Weiss, 2003); por ello, si consideramos el clima andino con alta nubosidad, se trata de una opción interesante. Colectores térmicos con fluido líquido suponen la recolección con placas y almacenamiento normalmente en tanque reservorio dispuesto 
verticalmente para aprovechar estratificación de refrigerante o agua caliente. En el tanque, la franja de almacenamiento con mayor temperatura (superior) se utiliza para ACS y el fluido en la franja inferior con menor temperatura contribuye a la calefacción, normalmente a través de sistema de baja temperatura como piso radiante. No obstante, la calefacción solar en países en desarrollo no se ha masificado por los costos de implementación y requerimiento de mayor superficie de recolección, además de la necesidad de tanque térmico con respaldo de calentamiento eléctrico con elevado volumen de almacenamiento. Las zonas ecuatoriales frías andinas serían un escenario ideal para su aplicación con colectores de tubo de vacío con alta capacidad ante irradiación difusa.

El abastecimiento eléctrico con captación PV sirve para suplir demandas eléctricas de iluminación, electrodomésticos, audio-video, etc. Residencialmente, la implementación más adecuada de colectores PV está en interconexión con la red pública, sobre todo en integración en vivienda, por cuanto las demandas normalmente no coinciden temporalmente con los máximos de producción solar, de modo que se prescinde de baterías de almacenamiento. Las instalaciones aisladas (off-grid) son una alternativa importante cuando la red está distante, sin embargo, el requerimiento de baterías y la limitada vida útil encarece el tipo de instalación. Aunque en el corto plazo se vislumbra la posibilidad de baterías eléctricas más eficientes, menos contaminantes, a precios accesibles y diseñadas para abastecer edificios (Tesla Inc., 2017).

Es posible, incluso, aportar electricidad contribuyendo a la red pública y demandas urbanas. No obstante, es necesaria una legislación que implique a las empresas eléctricas a comprar los excedentes a un precio razonable, incluso superior al precio de mercado (feed in tariff) para promover la masificación en etapas iniciales, a fin de lograr una reducción de costos hasta que la industria esté en capacidad de desarrollarse por sí sola (IEA, 2009; Lund, 2011). En varios lugares del mundo ya se han alcanzado precios PV inferiores al valor del mercado o grid parity, ofertándose en menos de 0,03 USD el Watt de Potencia en lugares de elevada irradiación, precios de producción muy inferiores a cualquier otro tipo de generación (Mahapatra, 2016). La desventaja financiera son los costos iniciales de instalación, son elevados a pagarse al principio, frente al precio normalmente accesible continuo a la compra directa a la red. 
Se recomienda emplazar los colectores PV sobre cámaras de aire ventiladas, la temperatura puede implicar una pérdida del $25 \%$ de eficiencia fácilmente al alcanzar los $70{ }^{\circ} \mathrm{C}$ (Agrawal y Tiwari, 2010; Hachem, 2012), más probable en días soleados y cálidos. Ello conlleva más posibilidades de integración en techumbres de viviendas al disponer PV que poseen las características de cubrimiento y capacidad de acoplarse, integrando en ellas sujeciones y resistencias apropiadas, incluso sellado en estanco. Se compone de una doble capa, la externa compuesta por colectores PV y una interna de fondo, la cual es capa estanca y de aislamiento. Entre las dos fluye ventilación para refrigeración, aire que una vez calentado puede contribuir a abastecer demandas térmicas como veremos en el siguiente apartado. Algunas investigaciones señalan que la mayor limitación para abastecer la demanda residencial en climas estacionales oscilantes es la alta demanda que implica calefacción o refrigeración extremas, factor que en zonas ecuatoriales no es una barrera por estabilidad climática, de demandas y de irradiación. Para calefacción con electricidad proveniente de PV o no, la alternativa es emplear Bomba de Calor de alta eficiencia (Athienitis, 2007).

\section{Abastecimiento térmico-eléctrico con captación solar híbrida}

La recolección solar híbrida posee la capacidad de entregar de modo simultáneo energía convertida en electricidad o en fluidos temperados para ACS o calefacción. Como es recomendable la ventilación de celdas fotovoltaicas, los colectores híbridos resultan de refrigeración requerida en PV y rescatan los remanentes térmicos. Existen dos tipos de colectores:

- Colectores híbridos con fluido líquido (BIPVTw): tienen aún baja industrialización, en consecuencia, todavía su precio es elevado lo que dificulta su aplicación masiva. No obstante, es una alternativa para el ámbito residencial, ya que entregan un rendimiento superior en exergía respecto a colectores PV o ST individualmente (Chow, 2010). Existen ya modelos que priorizan producción eléctrica o térmica, buscando adaptarse mejor a las demandas (Solimpeks, 2010).

- Colectores híbridos con fluido aire (BIPVTa: Building Integrated Photovoltaic \& Thermal air type). La refrigeración de las células tiene la capacidad de mejorar la producción eléctrica, con un incremento eléctrico suficiente para por lo menos energizar 
ventiladores que regulan la ventilación y ganancias térmicas adicionales. En zonas de muy baja irradiación se ha desestimado su funcionalidad por baja capacidad, no obstante, en latitudes medias-frías, con aceptable irradiación, es una opción rentable y está disponible comercialmente (Solarwall, 2015). La dificultad para su diversificación es la variación que debe existir en espesor de ducto de ventilación, porque para su máximo desempeño, tanto ancho como de longitud de cámara, debe estar en concordancia con el largo de faceta de techumbre y con irradiación directa adecuada (Agrawal y Tiwari, 2010; Astea, Del Peroa y Leonfortea, 2012; Athienitis et al., 2011).

\section{Criterios de integración arquitectónica}

Posturas de diseño respecto a la integración arquitectónica de colectores solares

Entre las estrategias que el arquitecto puede adoptar para integrar arquitectónicamente colectores solares, según Kaan y Reijenga (2004) están las siguientes posibilidades:

- Despliegue de modo que sean imperceptibles o invisibles, no denotan dónde empieza o dónde termina la superficie absorbente, no se identifican como colectores solares.

- Se suman e interponen al diseño original, normalmente estrategia práctica en restauraciones o renovaciones. Se busca uniformidad respecto al material y los ritmos modulares rítmicos con el edificio y sus componentes, mas no son elementos constitutivos y la edificación puede prescindir de ellos con afectaciones estructurales mínimas.

- Instalación de colectores con integración arquitectónica que influyen y contribuyen efectivamente en la apariencia del edificio, de manera intencionada.

- Colectores que determinan y conducen la imagen de la edificación.

- La captación solar activa se torna como aspecto prioritario en el diseño. Conjuntamente con la eficiencia energética, en el aspecto final de la edificación rigen las decisiones proyectuales.

\section{Tipos de adopción de colectores solares según el componente de envolvente recolectora}

Por último, los productos comerciales para integración arquitectónica, acordes con el componente de la envolvente que los incorpora, también pueden ser clasificables. Así, la Agencia 
Internacional de la Energía (IEA) define seis posibilidades morfológicas para integrarse arquitectónicamente (IEA y SHC Task 41, 2012a):

- Placas configuradas para techumbres inclinadas.

- Placas como cubierta horizontal en techumbres planas o cercanas a planas.

- Filtros solares de techumbre con la presencia de colectores como elementos de cobertura permeable, lo cual también puede provocarse en fachada.

- Ventanales fotovoltaicos o filtros solares para ventanas.

- Elementos compositivos de fachadas como balaustradas, sombrillas, filtros solares, etc., los cuales pueden ser colectores ST o PV.

- Recubrimiento o placas de fachada-envolvente, las cuales tienen capacidades aislantes y opacas, que son a la vez colectores ST o PV.

\section{Definición de niveles de integración arquitectónica de colectores}

Con base en las consideraciones previas y bajo análisis de los autores a los referentes de este trabajo, como compendio, se reconocen distintos niveles de inclusión de colectores considerando resultados expresivos, así como producción energética:

0 - (Sin integración): cuando los equipos de captación contribuyen a requerimientos energéticos, pero son independientes geométricamente (su forma no establece relación con alguna parte del edificio), y utilizan solamente la estructura existente como soporte físico (Terra Ecología Práctica, 2007).

1 - (Formal): los equipos poseen su superficie externa mayor en paralelo (y coplanar) a elementos regulares del edificio (fachadas o cubiertas), pero son prescindibles, se pueden retirar porque existe la superficie de cobertura subyacente (ver Auditorio Paolo VI en el Vaticano) (SUPSI Competence Center, 2008).

2 - (Funcional): los equipos poseen una superficie externa mayor en paralelo y coplanar, cumpliendo un rol de protección a la intemperie del edificio, como parte de la cubierta o 
revestimiento de fachada (ver productos Megasol® (Swiss Megasol y SUPSI Competence Center, 2011).

3 - (Expresión): los equipos poseen una superficie externa mayor que aporta al sentido expresivo general del edificio (ver edificio en van Heemskerckweg 9 en Venlo, Holanda) (Shade Optisol y SUPSI Competence Center, 2011).

4 - (Multiplicidad): los equipos aportan expresivamente y contribuyen de manera máxima a los requerimientos energéticos, rigiendo la expresión y forma final del edificio o, dicho de otro modo, el diseño del edificio toma la condicionante de "maximación energética" siendo prioridad en el diseño la eficiencia y producción energética así como su expresividad (ver The Eliotrope House) (Disch, 1994).

Los criterios mencionados no implican mejores resultados o una mejor alternativa por sí mismos entre niveles 1 y 4. La adhesión puede ser más oportuna, siempre y cuando sea considerado asertivamente respecto al diseño, o sea más adecuado que pasen imperceptibles, dependiendo del caso y del resultado. Aunque sí sería por lo menos cuestionable, desde la perspectiva arquitectónica el nivel 0 , "colectores simplemente colocados" en edificación, sin relación con aspectos arquitectónicos o del edificio en general (ver Figura 7, colectores sobrepuestos).

\section{Discusión}

Se han revisado distintos aspectos y criterios aún en desarrollo y teorización acerca de la inserción coherente de la recolección solar activa en arquitectura. La importancia de lograr a la brevedad sociedades, ciudades y edificios sostenibles es innegable; los edificios pueden contribuir a generar su propia energía, incluso produciendo excedentes para contribuir a demandas urbanas o externas bajo el Plus Energy Manifesto (Disch, 2010).

Adicional al diseño pasivo, como el aprovechamiento solar directo, la única manera de alcanzar edificaciones energéticamente neutras es la adopción de energías renovables conjuntamente. La opción solar es la alternativa idónea en lugares con elevada irradiación, aunque en zonas de latitudes altas, lejanas al ecuador, la integración en edificios de geotermia o minieólica ciertamente presentarían mejores posibilidades. 
Los colectores solares térmicos son tecnológicamente más sencillos, incluso los de fabricación artesanal, y son la opción para absorber demandas térmicas; en cuanto a los PV -hasta hace poco considerados de alto precio-, su desarrollo tecnológico, masificación e incremento en eficiencia han reducido dramáticamente sus costos, y su masificación en países en desarrollo es sustancial (Fraunhofer, 2016). Al punto de preverse que la fotovoltaica prevalecerá sobre la solar térmica gracias al almacenamiento de excedentes en red en Smart Grid de la totalidad de la generación, frente a las posibilidades de almacenamiento térmico que son limitadas, además de la versatilidad de la electricidad.

Respecto a eficiencia, los colectores térmicos con fluido líquido alcanzan un rendimiento de hasta el $80 \%$ de la radiación, y los térmicos con aire no sobrepasan el $50 \%$ (Kalogirou, 2004). Últimos avances muestran que la captación y conducción térmica por aire o refrigerante líquido permite una sencilla evacuación de excedentes térmicos que reducen la eficiencia de PV, por lo que la producción dual eléctrica-térmica también se prevé como una interesante opción (Hachem, Athienitis y Fazio, 2011). El almacenamiento en aire es limitado respecto a un medio líquido porque el calor específico del aire es inferior. Los fotovoltaicos, dependiendo la tecnología, ya alcanzan un $20 \%$ en productos comerciales, con tendencia creciente (Fraunhofer, 2016). Los colectores híbridos fotovoltaicos-térmicos refrigerados por aire alcanzan entre $29 \%$ hasta $42 \%$ de eficiencia térmica adicional a la eléctrica, aquellos con fluido de aire alcanzan acaso un mejor desempeño eléctrico respecto a colectores con fluido líquido por refrigerar mejor las celdas y no requerir una capa de acristalamiento para conformar efecto invernadero dentro del colector. Comparativamente, en el desempeño térmico de colector híbrido (BIPVTw) respecto al colector térmico tipo placa plana (Tw) se ha encontrado una penalización de eficiencia de entre $29 \%$ con baja refrigeración hasta $50 \%$ con alta refrigeración.

El calor conducido por aire se puede almacenar en masa térmica en los propios elementos constructivos de la edificación o con intercambiador aire-agua para colaborar con el agua caliente sanitaria (ACS). Los captadores híbridos con fluido aire se han descartado en climas de irradiación baja; no obstante, poseen potencial en zonas meridionales a partir de una latitud semejante a la franja sur de Canadá $(45 \mathrm{~s} \mathrm{~N})$, en donde se ha implementado con éxito y es distribuido comercialmente (Solarwall, 2015). Se supone una relación de producción térmica/eléctrica igual o superior a 3 (Hachem, 2012) no obstante, las ganancias térmicas no son 
útiles siempre, ya que son excesivas en verano, de allí la importancia de contribuir con ACS en periodos de alta radiación, a través de integrarse con un intercambiador de calor de fluido aire a líquido.

En cuanto a consideraciones arquitectónicas y posturas de diseño, existen ya en el mercado colectores (aunque aún en proceso de desarrollo) con capacidad de incorporarse en el edificio y pasar desapercibidos. La perspectiva quizás opuesta es buscar un resultado expresivo del edificio consecuente con la expresión misma de los colectores desplegados, potenciando su expresión. Entre los dos extremos existen posturas medias. No es posible definir de manera absoluta cuál es más adecuada, pues, por ejemplo, un edificio patrimonial emplazado en un contexto histórico es distinto a uno localizado en un lugar sin valor especial. En cuanto a los niveles de integración propuestos por los autores, el nivel 0 vendría a representar la no consideración arquitectónica aspectual del despliegue de los colectores como resultado de la colocación de los sistemas solares por mejor captación o facilidad de instalación, postura que afecta definitivamente el aspecto arquitectónico; el otro nivel extremo (Nivel 4) sería cuando el edificio alcanza a maximizar la producción energética y ello influye en la arquitectura, siendo esta la consecuencia de la eficiencia energética. Por último, la aplicación de los colectores puede darse básicamente en cualquier elemento envolvente, tanto techos como muros exteriores, además parasoles, balaustradas, incluso ventanas transparentes o traslúcidas, en este último caso es desarrollo resiente y notable con posibilidades de filtrar el exceso de irradiación y rayos UV generando a la vez electricidad (OnyxSolar, 2017). La adopción de cualquiera de estos elementos como recolector solar debería también ser el resultado de una decisión arquitectónica oportuna.

\section{Conclusiones}

Se ha hecho una revisión de las posibilidades de aprovechamiento solar en la arquitectura y el desarrollo de sistemas solares activos y su potencial para reducir las demandas energéticas en edificación. Se han revisado las posibilidades tecnológicas existentes, planteamientos teóricos de posturas que el arquitecto puede adoptar frente a la adopción de estos sistemas tecnológicos, y se reconocen las limitaciones y necesidades para que en el futuro se desplieguen masivamente en integración arquitectónica. 
Se concluye que no es posible establecer reglas absolutas acerca de las características técnicas, morfológicas y de adaptabilidad para la instalación de recolección solar activa, más bien deben estar en correspondencia con condiciones puntuales, siendo muy relativo; en ello el papel decisivo del arquitecto es fundamental. El arquitecto debe conocer, aunque de manera básica, las tecnologías disponibles, correlación con demandas, conocimiento de herramientas proyectuales con posibilidades de aplicarse en etapas tempranas de proyecto; además, sería deseable tener accesibilidad a variedad de productos de integración arquitectónica.

Bajo esta perspectiva, se han enumerado aspectos teóricos acerca del concepto de integración solar activa, determinando consideraciones estéticas, técnicas y funcionales. Solamente con la integración desde etapas tempranas de diseño es factible lograr una adaptación coherente en la envolvente que responda a aspectos de disposición espacial, orientación, en concordancia con demandas o criterios de acoplamiento estructural y, finalmente, en relación con consideraciones aspectuales y estéticas. Todas estas consideraciones no son absolutas ni globalizables, porque los parámetros mencionados varían según las realidades locales, constructivas y funcionales, por lo que cada situación exige estudio y soluciones particulares. Los productos tampoco se desempeñan de igual manera en todos los niveles de radiación, las demandas también son determinantes. Este trabajo ha tratado de cubrir las principales condiciones.

Este trabajo propone, a partir del referente Niveles de Integración, para sumarse a la discusión actual, una gradación de integración, aunque resaltando que un Nivel elevado (4) no significa de manera absoluta un mejor resultado, sobre un Nivel 1; este dependerá del caso y de la implicación del impacto. Aunque desde nuestra perspectiva, la postura que adopte el arquitecto se verá enmarcada en algún nivel, dependiendo de la solución que escoja al integrar colectores en la envolvente.

Por último, es necesario reconocer que los productos para integración arquitectónica están aún en desarrollo, la disponibilidad de productos para reemplazar elementos constructivos o constitutivos en una edificación aún no se alcanza a escala comercial, lo que es una labor pendiente desde la industria. Si bien hay productos comerciales desarrollados, estos son difíciles de ser reemplazados por desperfectos o mantenimiento ya que cada industria fabrica distintos modelos no estandarizados. Acaso la estandarización en modulación y tecnología es necesaria. 
Por ahora, el crecimiento en la incorporación de fotovoltaicos y térmicos es sustancial, alcanzando ya en Estados Unidos un millón de casas solamente con PV y en crecimiento, pero con productos no integrados sino sobrepuestos por las dificultades técnicas de los productos integrados (Cinnamon, 2016). Es necesario, entonces, un abordaje más incisivo desde la arquitectura. Aunque los colectores para integración arquitectónica desarrollados hasta la actualidad muestran en su mayoría tendencia a mimetizarse en el aspecto del edificio, también existe la postura de la autenticidad, es decir, que los colectores deben mostrarse y resaltarse como tales, y no "asemejarse" a revestimientos típicos como tejas o placas. Esta autenticidad conlleva ventajas técnicas como mayor eficiencia, y muestra preocupación por el medio ambiente.

Bajo cualquier paradigma adoptado por parte de los diseñadores, es necesario que la disciplina considere y demande incorporar estos sistemas, así existirá demanda a la industria, con el subsecuente desarrollo de los sistemas y la consecuente reducción de precios.

\section{Referencias Bibliográficas}

Agrawal, B. y Tiwari, G. N. (2010). Optimizing the energy and exergy of building integrated photovoltaic thermal (BIPVT) systems under cold climatic conditions. Appl. Energy, 87, 417 426. doi:10.1016/j.apenergy.2009.06.011

Alamy (2015). Impact 2000 House. Recuperado de http://www.alamy.com/stock-photo-pvhouse-the-boston-edison-impact-2000-home-incorporated-a-4-kwp-utility-84599920.html

Astea, N., Del Peroa, C. y Leonfortea, F. (2012). Optimization of solar thermal fraction in PVT systems. Energy Procedia, 30, 8-18. doi:10.1016/j.egypro.2012.11.003

Athienitis, A. K. (2007). Design of a solar home with bipv-thermal system and ground source heat pump. Canadian Solar Buildings Conference, Calgary.

Athienitis, A. K., Bambara, J., ONeill, B. y Faille, J. (2011). A prototype photovoltaic/thermal system integrated with transpired collector. Sol. Energy 117, 403-410. doi:10.1016/j.solener.2010.10.008

Besser, D., Rodrigues, L. y Bobadilla, A. (2012). New Chilean Building Regulations and Energy Efficient Housing in Disaster Zones The thermal performance of prefabricated timber-frame 
dwellings. PLEA 2012 - 28th Conf. Oppor. Limits Needs Towar. An Environ. Responsible Archit.

Bolinger, M. y Wiser, R. (2002). Case studies of state support for renewable energy. Estados Unidos: Berkeley Lab and the Clean Energy Group CASE.

CDT (2007). Sistemas Solares Térmicos. Santiago de Chile: Corporación de Desarrollo Tecnológico.

Chow, T. T. (2010). A review on photovoltaic/thermal hybrid solar technology. Appl. Energy, 87, 365-379. doi:10.1016/j.apenergy.2009.06.037

Cinnamon, B. (2016). When Can I Get Solar Shingles? San Diego: Cinnamonsolar.

De Little, A. (1995). Building- Integrated Photovoltaics and US Market. Massachusets: U.S. Department of Energy.

Disch, R. (2010). PlusEnergy - The Manifesto. Recuperado de http://www.rolfdisch.de/files/pdf/12_PLUSENERGIE_EIN_MANIFEST_6_englisch.pdf.

Disch, R. (1994). Rotatable Solar House HELIO-TROP ®. Freiburg: Rolf Disch SolarArchitektur.

EREC (2010). RE-thinking 2050: a 100\% renewable energy vision for the European Union. Bruselas: EREC.

Fraunhofer, Institute for Solar Energy (2016). Photovoltaics report. Freiburg: Fraunhofer Institute for Solar Energy Systems, ISE

Gajbert, H. (2008). Solar thermal energy systems for building integration. Lund: Lund University.

Gook-hwan, H. y Eximbank, K. (2013). Smart Grid Studies in Ecuador. Sejong: Knoeledge Sharing Program Korea

Guillén, V., Quesada, F., López, M., Orellana, D., Serrano, A., Mena, V.G. et al. (2014). Eficiencia energética en edificaciones residenciales. ESTOA, 63-73. 
Gupta, A., Cemesova, A., Hopfe, C. J., Rezgui, Y. y Sweet, T., (2014). A conceptual framework to support solar PV simulation using an open-BIM data exchange standard. Autom. Constr. 37, 166-181. doi:10.1016/j.autcon.2013.10.005

Haberl, J. S. y Cho, S. (2014). Energy Systems. Work. Gr. III - Mitig. Clim. Chang., 139.

Hachem, C. (2012). Investigation of Design Parameters for Increased Solar Potential of Dwellings and Neighborhoods. Montreal: Concordia University.

Hachem, C., Athienitis, A. y Fazio, P (2011). Investigation of solar potential of housing units in different neighborhood designs. Energy Build, 43, 2262-2273. doi:10.1016/j.enbuild.2011.05.008 IEA (2009). Cities, Towns \& Renewable Energy Cities, Towns. Paris: IEA/OECD.

IEA y SHC (2015). New Generation Solar Cooling \& Heating Systems. Recuperado de URL http://task53.iea-shc.org.

IEA y SHC (1977). IEA Solar Heating \& Cooling Programme. Recuperado de http://www.ieashc.org/tasks-completed.

IEA y SHC (2014). Innovative solar products for building integration Recuperado de http://www.solarintegrationsolutions.org

IEA y SHC Task 26 (2000). CombiSystem Overview 2000. Recuperado de http://www.aeeintec.at/0uploads/dateien551.pdf

IEA y SHC Task 41 (2012a). Solar energy systems in architecture, integration criteria and guidelines. Recuperado de http://task41.iea-shc.org/data/sites/1/publications/T41DA2-SolarEnergy-Systems-in-Architecture-28March2013.pdf

IEA y SHC Task 41 (2012b). Solar design of buildings for architects: Review of solar design tools. Recuperado de http:/task41.iea-shc.org/data/sites/1/publications/T41 DA2-Solar-EnergySystems-in-Architecture-28March2013.pdf 
IEA Solar Heating y Cooling Program (2007). Compilation and analyze of interviews DA 1-2 Preliminary Outcome of PV / T market survey interviews. Härnösand: IEA Solar Heating and cooling Program.

IEA Solar Heating y Cooling Program Task 16 (1995). Photovoltaic in Buildings. Recuperado de http://archive.iea-shc.org/task16/index.html

Kaan, H. y Reijenga, T. (2004). Photovoltaics in an architectural context. Prog. Photovoltaics Res. Appl., 12, 395-408. doi:10.1002/pip.554

Kalogirou, S. A. (2004). Solar thermal collectors and applications. Prog. Energy Combust. Sci., 30, 231-295. doi:10.1016/j.pecs.2004.02.001

Lamnatou, C., Mondol, J. D., Chemisas-bipv-onyxsolar.html

Lorenzo, E., 2004. Pioneros españoles de las energías renovables Introducción, in: XII Congreso Iberico Y VII Americano de Energía Solar.

Lund, P., 2012. Large-scale urban renewable electricity schemes - Integration and interfacing aspects. Energy Convers. Manag. 63, 162-172. doi:10.1016/j.enconman.2012.01.037

Lund, P., 2011. Boosting new renewable technologies towards grid parity - Economic and policy aspects. Renew. Energy 36, 2776-2784. doi:10.1016/j.renene.2011.04.025

Mahapatra, S., 2016. Lowest-Ever Solar Price Bid $(2.42 \varnothing / \mathrm{kWh})$ Dropped In Abu Dhabi By JinkoSolar \& Marubeni Score [WWW Document]. Cleantechnica. URL https://cleantechnica.com/2016/09/20/lowest-ever-solar-price-bid-2-42 фkwh-dropped-abu-dhabijinkosolar-marubeni-score/

Menayo, A., 2010. Carcaterización de la Cocina Solar Primrose. Madrid : Carlos III de Madrid.

Mikkola, J., Lund, P.D., 2014. Models for generating place and time dependent urban energy demand profiles. Appl. Energy 130, 256-264. doi:10.1016/j.apenergy.2014.05.039

Ministerio de Energía de Chile (2010). Norma técnica que determina el algoritmo para la verificación de la contribución solar mínima de los sistemas solares térmicos acogidos a la franquicia tributaria de la Ley 20.365. Chile: Ministerio de Energía de Chile. 
Munari, C., 2009. Architectural Integration and Design of Solar Thermal Systems. ÉCOLE POLYTECHNIQUE FÉDÉRALE DE LAUSANNE.

Naranjo-Mendoza, C., Rousse, D.R., Quesada, G., 2013. Modeling of a solar absoprtion cooling system for Guayaquil, Ecuador, in: 2nd International Conference on Renewable Energy Research and Applications (Ed.), . Madrid, pp. 853-856. doi:10.1109/ICRERA.2013.6749870

Natural Resources Canada, 2016. RETScreen Clean Energy Management Software [WWW Document]. URL http://www.nrcan.gc.ca/energy/software-tools/7465 (accessed 5.12.16).

OnyxSolar, 2017. Soluciones BIPV [WWW Document]. URL http://www.onyx solar.com/es/soluciones-bipv-onyxsolar.html (accessed 4.16.17).

Perlin, J. (2013). Let It Shine: The 6000 Year Story of Solar Energy. Recuperado de http://johnperlin.com/let-it-shine.html

Rickerson, W. e IEA (2014). Residential prosumers - drivers and policy options. Re-prosumers, 1-123.

Shade Optisol y SUPSI Competence Center (2011). Detail sheet Solar shadings. Cannobio: Swiss BiPV Competence Centre.

Solar Design Associates (2015). Carlisle House. Recuperado de http://www.solardesign.com/SDA_Today/carlisle-house/

Solarwall (2015). PV/Thermal; Hybrid Solar Heating + Electricity. Recuperado de http://solarwall.com/en/products/pvthermal.php

Solimpeks (2010). Volther Hybrid PV-T Panels. Konya: Solimpeks Solar Energy Corporation.

SUPSI Competence Center (2008). Paolo VI Audience Chamber. Canobbio: Swiss BiPV Competence Centre.

Suter, J.-M., Letz, T. y Weiss, W. (2003). Solar Combisystems - Overview. Gleisdorf: AEE INTEC.

Swiss Megasol y SUPSI Competence Center (2011). Façade elements Megasol Swiss Premium Mono. Canobbio: Swiss BiPV Competence Centre. 
Terra Ecología Práctica (2007). Guía práctica de una instalación de energía solar térmica. Recuperado de http://www.terra.org/categorias/articulos/guia-practica-de-una-instalacion-deenergia-solar-termica.

Tesla Inc. (2017). PowerWall Tesla Home Battery. Recuperado de http://www.teslamotors.com/powerwall

Vázquez Espí, M. (1999). Una brevísima historia de la arquitectura solar. Por una Arquitectura y un Urbanismo Contemporáneos, 1-31.

Wall, M., Munari Probst, M. C., Roecker, C., Dubois, M. C., Horvat, M., Jorgensen, O. B. y Kappel, K. (2012). Achieving solar energy in architecture - IEA SHC Task 41. Energy Procedia, 30, 1250-1260. doi:10.1016/j.egypro.2012.11.138

Wegertseder, P., Lund, P., Mikkola, J. y García Alvarado, R. (2016). Combining solar resource mapping and energy system integration methods for realistic valuation of urban solar energy potential. Sol. Energy, 135, 325-336. doi:10.1016/j.solener.2016.05.061

Zalamea León, E. y García Alvarado, R. (2014). Integrated architectural design of active solar thermal collector at dwelling's roofs. Arquitectura y Urbanismo, XXXV, 1815-5898.

Zhu, H., Wei, J., Wang, K., Wu, D., Al-Hasan, A. Y., Altermatt, P P et al. (2011). The history of solar. Sol. Energy Mater. Sol. Cells, 93, 1461-1470. doi:10.1016/j.solmat.2009.04.006: 